University of South Carolina

Scholar Commons

$5-15-2011$

\title{
Spontaneous Emergence of a Persistent Spin Helix from Homogeneous Spin Polarization
}

\author{
Valeriy A. Slipko \\ Ibrahim Savran \\ Yuriy V. Pershin Dr \\ University of South Carolina - Columbia, pershin@physics.sc.edu
}

Follow this and additional works at: https://scholarcommons.sc.edu/phys_facpub

Part of the Physics Commons

\footnotetext{
Publication Info

Published in Physical Review B, ed. Gene D. Sprouse, Volume 83, Issue 19, 2011, pages 193302-1-193302-4.

Slipko, V. A., Savran, I., \& Pershin, Y. V. (2011). Spontaneous emergence of persistent spin helix from homogeneous spin polarization. Physical Review B, 83(19), 193302-1 - 193302-4. DOI: 10.1103/

PhysRevB.83.193302

(c) Physical Review B, 2011, American Physical Society accepted for inclusion in Faculty Publications by an authorized administrator of Scholar Commons. For more information, please contact digres@mailbox.sc.edu.
} 


\title{
Spontaneous emergence of a persistent spin helix from homogeneous spin polarization
}

\author{
Valeriy A. Slipko, ${ }^{1,2}$ Ibrahim Savran, ${ }^{3}$ and Yuriy V. Pershin ${ }^{1, *}$ \\ ${ }^{1}$ Department of Physics and Astronomy and USC Nanocenter, University of South Carolina, Columbia, South Carolina 29208, USA \\ ${ }^{2}$ Department of Physics and Technology, V. N. Karazin Kharkov National University, Kharkov 61077, Ukraine \\ ${ }^{3}$ Department of Computer Science and Engineering, University of South Carolina, Columbia, South Carolina 29208, USA
}

(Received 31 January 2011; revised manuscript received 21 March 2011; published 16 May 2011)

\begin{abstract}
We demonstrate that a homogeneous spin polarization in one-dimensional structures of finite length in the presence of Bychkov-Rashba spin-orbit coupling decays spontaneously toward a persistent spin helix. Such a strikingly different and simple method enables us to generate robust spin structures whose properties can be tuned by the strength of the spin-orbit interaction and/or the structure's length. We generalize our results for the two-dimensional case predicting the formation of a persistent spin helix in two-dimensional channels from homogeneous spin polarization. An analysis of the formation of a spin helical state is presented within an approach based on a mapping of spin drift-diffusion equations into a heat equation for a complex field.
\end{abstract}

A helical wave of rotating spin orientation is referred to as a spin helix. There is a significant interest in spin helix configurations in semiconductor materials since the electron spin relaxation of such spin configurations can be partially ${ }^{1-3}$ or even completely suppressed..$^{4,5}$ While a partial suppression of spin relaxation in two-dimensional systems becomes possible in the presence of only Bychkov-Rashba ${ }^{6}$ spin-orbit coupling (see Refs. 1 and 2), the complete suppression of spin relaxation requires a specific combination of Bychkov-Rashba and Dresselhaus ${ }^{7}$ interactions, as was demonstrated in Refs. 4 and 8. More generally, the relaxation of the spin helix is an example of situations ${ }^{1-5,9-18}$ in which the electron spin relaxation scenario deviates from the predictions of D'yakonov-Perel' theory. ${ }^{19}$

Experimentally, the spin-grating technique ${ }^{20}$ is typically used $^{3,5}$ to create spin helical configurations in semiconductors. In this method, a sample is illuminated by a pair of pump beams with orthogonal linear polarizations. The interference of such beams results in a spacial modulation of light helicity. Correspondingly, through the optical orientation effect, a modulation of spin polarization in the form of a spin helix is produced. Moreover, a spin injection from a ferromagnetic material into a semiconductor can also be used to excite a spin helix. ${ }^{8}$ In this approach, the rotating spin polarization is caused by coherent spin precession of electrons drifting in an applied electric field. However, we are not aware of any experimental studies on spin helixes excited by spin injection.

In this Brief Report, we propose an alternative approach to induce spin helical configurations. Specifically, we demonstrate that in one-dimensional (1D) systems of finite length with Bychkov-Rashba spin-orbit coupling, the spin helical configurations emerge in the process of relaxation of homogeneous spin polarization (see Fig. 1). Mathematically, such a strikingly unexpected transformation of homogeneous spin polarization into the persistent spin helix occurs when we introduce boundary conditions on electron space motion to describe finite-length structures (in infinite systems, the homogeneous spin polarization decays exponentially, as predicted by D'yakonov-Perel' theory ${ }^{19}$ ). Using an approach that maps spin drift-diffusion equations into a heat transfer equation for a complex field, we find the exact time dependence of the spin-polarization dynamics. It is interesting that the amplitude of the resulting spin helix has an oscillatory dependence on the system's length. Below, we provide an intuitive explanation of this result based on the properties of the solution of the heat equation. Moreover, it is necessary to emphasize that our theory is generalized for the case of two-dimensional (2D) channels and can be straightforwardly verified experimentally. In particular, experimentally, the homogeneous spin polarization can be easily created using the optical orientation by circularly polarized light. Therefore, we believe that our approach would simplify tremendously the generation of longliving spin helical configurations in semiconductor structures and advance the field of spin storage in semiconductors.

Let us consider the dynamics of electron spin polarization in a 1D system of length $L$ in the $x$ direction in the presence of Bychkov-Rashba spin-orbit coupling. In the one-dimensional limit, spin drift-diffusion equations ${ }^{2}$ (see also Refs. 21 and 22) can be written as

$$
\begin{gathered}
\frac{\partial S_{x}}{\partial t}=D \Delta S_{x}+C \frac{\partial S_{z}}{\partial x}-2 \gamma S_{x}, \\
\frac{\partial S_{y}}{\partial t}=D \Delta S_{y}, \\
\frac{\partial S_{z}}{\partial t}=D \Delta S_{z}-C \frac{\partial S_{x}}{\partial x}-2 \gamma S_{z},
\end{gathered}
$$

where $D=\ell^{2} / \tau$ is the coefficient of diffusion, $\Delta=\partial^{2} / \partial x^{2}$, $C=2 \eta D$ is the constant describing spin rotations, $\gamma=\eta^{2} D / 2$ is the coefficient describing spin relaxation, $\eta=2 \alpha m \hbar^{-1}$ is the spin precession angle per unit length, $\alpha$ is the spin-orbit coupling constant, $m$ is the effective electron mass, $\ell$ is the mean free path, and $\tau$ is the momentum relaxation time. It follows from Eq. (2) that the $y$ component of spin polarization, $S_{y}$, is not coupled to any other component of spin polarization. Consequently, selecting $S_{y}(x, t=0)=0$, we can safely take out $S_{y}$ from our consideration. Equations (1) and (3) are complimented by standard boundary conditions ${ }^{23}$

$$
\left(2 D \frac{\partial S_{x}}{\partial x}+C S_{z}\right)_{\Gamma}=0,\left(2 D \frac{\partial S_{z}}{\partial x}-C S_{x}\right)_{\Gamma}=0 .
$$

Here, $\Gamma=[x=0, x=L]$. Mathematically, the boundary conditions (4) are so-called third-type boundary conditions. This specific form of boundary conditions conserves the spin polarization of electrons that scatter from the sample edges. We 


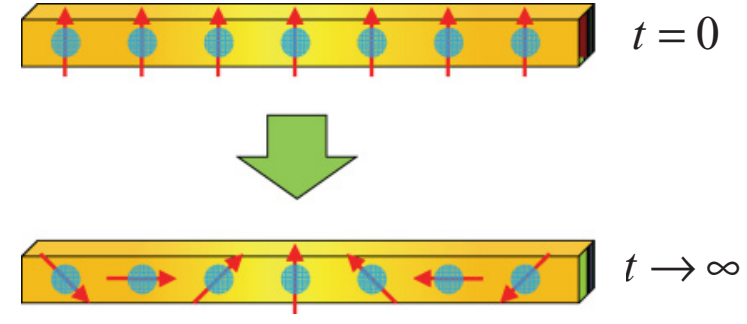

FIG. 1. (Color online) Schematics of spontaneous transformation of homogeneous spin polarization into a persistent spin helix in a finite length system with Bychkov-Rashba spin-orbit coupling.

assume that at the initial moment of time the spin polarization is homogeneous and points in the $z$ direction, that is,

$$
S_{x}(x, t=0)=0, S_{z}(x, t=0)=S_{0} .
$$

We note that although Eq. (5) does not satisfy Eq. (4), that does not create a problem since the diffusion-type equations (1)-(3) with boundary conditions (4) are stable. We could also modify initial conditions (5) by an infinitely small correction near the boundaries that would make them satisfy Eq. (4). However, the solution with modified initial conditions would be infinitely close to the solution with initial conditions (5).

Let us introduce a complex polarization $S=S_{x}+i S_{z}$. It is straightforward to show that Eqs. (1) and (3) and boundary conditions (4) can be rewritten in a more compact form using $S$ :

$$
\begin{gathered}
\frac{\partial S}{\partial t}=D \frac{\partial^{2} S}{\partial x^{2}}-i C \frac{\partial S}{\partial x}-2 \gamma S, \\
\left(2 D \frac{\partial S}{\partial x}-i C S\right)_{\Gamma}=0 .
\end{gathered}
$$

Defining a complex field $u(x, t)$ by the relation

$$
u(x, t)=e^{-i \eta x} S(x, t),
$$

we find that Eq. (6) transforms into the heat equation

$$
\frac{\partial u}{\partial t}=D \frac{\partial^{2} u}{\partial x^{2}},
$$

supplemented by Neumann (or second-type) boundary conditions

$$
\left(\frac{\partial u}{\partial x}\right)_{\Gamma}=0
$$

Moreover, it is worth noticing that the initial conditions for $u(x, t)$ are related to the initial conditions for $S$ as

$$
u(x, t=0)=e^{-i \eta x} S(x, t=0) .
$$

Consequently, the initially homogeneous spin polarization in the $z$ direction [Eq. (5)] corresponds to a spatially modulated complex field

$$
u(x, t=0)=S_{0} \sin (\eta x)+i S_{0} \cos (\eta x) .
$$

The solution of Eq. (9) with the boundary conditions (10) and initial condition (12) was obtained by the method of separation of variables. It can be presented in the form

$$
\begin{aligned}
\frac{S(x, t)}{S_{0}}= & i \frac{\sin (\eta L / 2)}{\eta L / 2} e^{i \eta(x-L / 2)}+2 \eta L e^{i \eta x} \\
& \times \sum_{n=1}^{+\infty} \frac{1-(-1)^{n} e^{-i \eta L}}{(\eta L)^{2}-(\pi n)^{2}} e^{-\frac{\pi^{2} n^{2} D t}{L^{2}}} \cos \left(\frac{\pi n x}{L}\right) .
\end{aligned}
$$

This is our main analytical result describing the dynamics of spin polarization in 1D finite-length structures. Note that $S_{x}$ and $S_{z}$ components of spin polarization are given by real and imaginary parts of Eq. (13), respectively. The first term on the right-hand side of Eq. (13) describes the persistent profile of spin polarization (in the form of a spin helix) emerging at long times. Concerning the second term on the right-hand side of Eq. (13), it governs the dynamics of transformation of the initially homogeneous spin polarization into the persistent spin helix. Figure 2 demonstrates the dynamics of $S_{z}$ and $S_{x}$ components of spin polarization given by Eq. (13). It is clearly seen that the initially homogeneous spin polarization in the $z$ directions transforms into the persistent spin helix with an (infinitely) long lifetime.

Explicitly, in the long-time limit, the spin polarization is given by

$$
\begin{gathered}
S_{x}(x, t=+\infty)=-S_{0} \frac{\sin (\eta L / 2)}{\eta L / 2} \sin [\eta(x-L / 2)], \\
S_{z}(x, t=+\infty)=S_{0} \frac{\sin (\eta L / 2)}{\eta L / 2} \cos [\eta(x-L / 2)] .
\end{gathered}
$$

In these equations, the factor $\sin (\eta L / 2) /(\eta L / 2)$ defines reduction of the spin helix amplitude with respect to the initial amplitude of homogeneous spin polarization $S_{0}$. We plot this function in Fig. 3. It is interesting that the spin helix amplitude is an oscillating function of the parameter $\eta L$ and takes zero values when $\eta L=2 \pi n$, where $n$ is a positive integer. The positions of local maxima can be found numerically. In particular, the positions of four local maxima shown in Fig. 3 are $8.987,15.450,21.808$, and 28.132 .

The heat equation is the best starting point to understand the oscillatory dependence of spin helix amplitude on $\eta L$ depicted in Fig. 3. According to Eq. (11), the initially homogeneous initial condition [Eq. (5)] for spin-diffusion equations transforms into a modulated initial condition for the heat equation. As the solution of the heat equation in the given context represents simply the process of "temperature" equilibration along the system, an integer number of modulation periods results in zero average "temperature" and, correspondingly, in zero spin helix amplitude. Moreover, we would like to mention that the spin helix formation process is described by a series of exponentially decaying terms whose time constants are given by $\tau_{n}=L^{2} /\left(\pi^{2} n^{2} D\right)$. The longest of these times $\tau_{1}=L^{2} /\left(\pi^{2} D\right)$ provides the time scale of the transformation process. The dependence of the longest spin-relaxation time on $L$ is intuitively clear as the characteristic relaxation time scale is determined by the time it takes for an electron to diffusively propagate over the wire length $L$. It is also interesting that such a time can be longer or shorter than the relaxation time of homogeneous spin polarization $\tau_{h}=1 /\left(D \eta^{2}\right)$. In particular, 

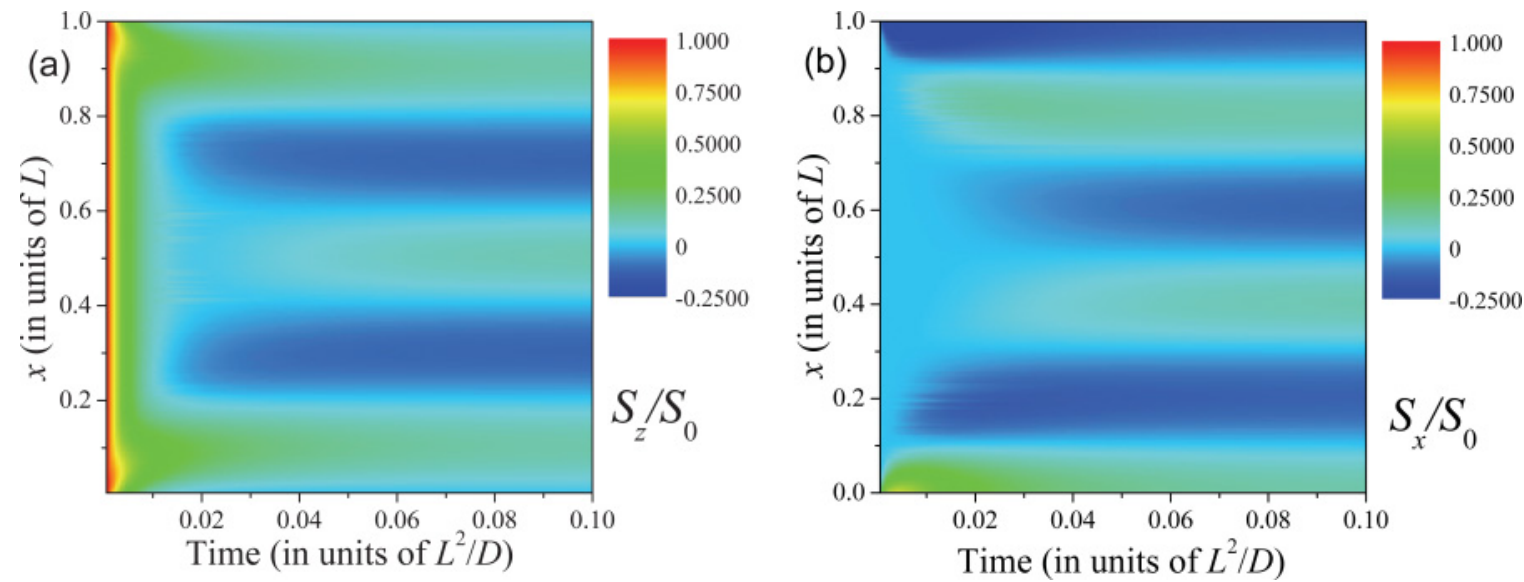

FIG. 2. (Color online) Dynamics of the formation of a persistent spin helix from homogeneous spin polarization pointing in the $z$ direction at $t=0$. These plots were obtained using Eq. (13) at $\eta L=15.45$. This value of the parameter $\eta L$ corresponds to the second local maximum of spin helix amplitude shown in Fig. 3.

$\tau_{1} / \tau_{h}=(\eta L / \pi)^{2}$, meaning that $\tau_{1}<\tau_{h}$ when $\eta L<\pi$, the times are the same when $\eta L=\pi$, and $\tau_{1}>\tau_{h}$ when $\eta L>\pi$ (see also Fig. 3).

To obtain additional insight into the spin relaxation of the spin helix, we have performed extensive Monte Carlo simulations employing an approach described in Refs. 9 and 24. This Monte Carlo simulation method uses a semiclassical description of electron space motion and a quantummechanical description of spin dynamics (the latter is based on the Bychkov-Rashba coupling term). All specific details of the Monte Carlo simulations program can be found in the references cited above and will not be repeated here. A spin conservation condition was used for electrons scattering from system boundaries. Generally, all obtained Monte Carlo simulation results are in perfect quantitative agreement with our analytical predictions, thus confirming the mechanism of

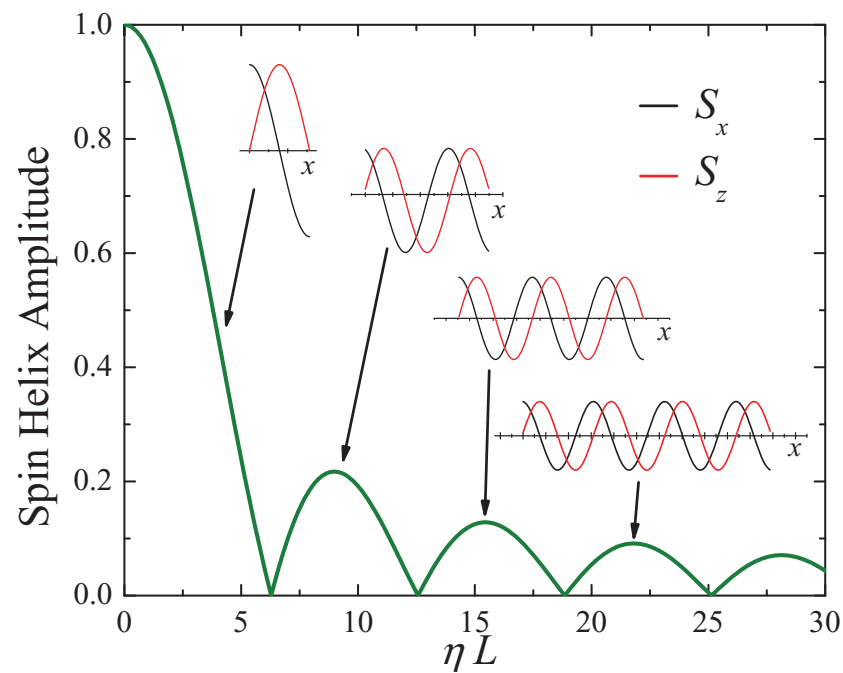

FIG. 3. (Color online) Normalized amplitude of the persistent spin helix as a function of $\eta L$. Insets show schematically distributions of $S_{x}$ and $S_{z}$ at several specific values of $\eta L$ as indicated by arrows. Positions of minima and maxima points of the amplitude are discussed in the text. the formation of a persistent spin helix from homogeneous spin polarization. A comparison of selected analytical and numerical curves is given in Fig. 4.

The results reported in this paper can be readily generalized for the persistent spin helix in two dimensions. ${ }^{4}$ Indeed, it can be easily seen that in the case of equal strength of Bychkov-Rashba and Dresselhaus spin-orbit interactions, $\alpha=$ $\beta$ (where $\beta$ is the Dresselhaus spin-orbit coupling constant), the equations of spin diffusion in $2 \mathrm{D}^{4}$ take the general form of Eqs. (1)-(3). Therefore, introducing appropriate boundary conditions, namely, reducing the system into a $2 \mathrm{D}$ channel in the [-110] direction (see the inset in Fig. 4), we obtain a situation completely equivalent to that in $1 \mathrm{D}$ from the point of view of spin dynamics. Taking into account a recent experimental demonstration of a persistent spin helix, ${ }^{5}$ the

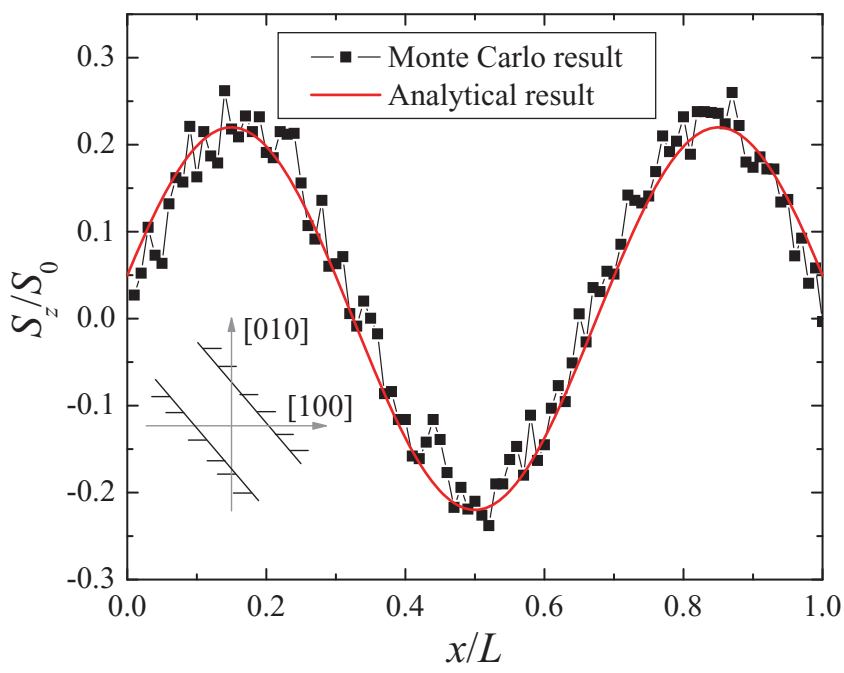

FIG. 4. (Color online) Long-time distribution of $S_{z}$ at $\eta L=$ 8.987 found employing the Monte Carlo simulation approach. The analytical curve is obtained using Eq. (15). The Monte Carlo simulation was performed for $10^{5}$ electrons in a GaAs structure of $1.7 \mu \mathrm{m}$ length. This plot was obtained using the parameter values $\tau=0.1 \mathrm{ps}, l=10 \mathrm{~nm}, \alpha=3 \times 10^{-12} \mathrm{eV} \mathrm{m} / \hbar$. Inset: orientation of the $2 \mathrm{D}$ channel for $2 \mathrm{D}$ spin helix excitation experiments. 
emergence of a persistent spin helix from homogeneous spin polarization can be detected straightforwardly. Finally, we would like to note that the amplitude of a persistent spin helix can be increased by a repetitive excitation of homogeneous polarization by a train of laser pulses.

In summary, we have demonstrated that a persistent spin helix forms in the process of relaxation of homogeneous spin polarization in finite length systems. This observation can be used as a different technique for creating spin helical structures in semiconductors. The solution of spin drift-diffusion equations describing the formation of a persistent helix was derived analytically and numerically using the Monte Carlo simulation approach. The results obtained using both methods are in perfect agreement. It is interesting that the persistent helix amplitude demonstrates an oscillatory dependence on the system length and strength of spin-orbit interaction. Therefore, the control of spin helix characteristics is achievable via an appropriate choice of the above-mentioned parameters. This suggested technique facilitates the generation of spin helical states and can be used in both one- and two-dimensional geometries.

I.S. acknowledges financial support from the Republic of Turkey Ministry of National Education, Grant No. MEB1416. *pershin@physics.sc.edu

${ }^{1}$ Y. V. Pershin, Phys. Rev. B 71, 155317 (2005).

${ }^{2}$ Y. V. Pershin and V. A. Slipko, Phys. Rev. B 82, 125325 (2010).

${ }^{3}$ C. P. Weber, J. Orenstein, B. A. Bernevig, S.-C. Zhang, J. Stephens, and D. D. Awshalom, Phys. Rev. Lett. 98, 076604 (2007).

${ }^{4}$ B. A. Bernevig, J. Orenstein, and S.-C. Zhang, Phys. Rev. Lett. 97, 236601 (2006).

${ }^{5}$ J. D. Koralek, C. P. Weber, J. Orenstein, B. A. Bernevig, S.-C. Zhang, S. Mack, and D. D. Awschalom, Nature (London) 458, 610 (2009).

${ }^{6}$ Y. A. Bychkov and E. I. Rashba, JETP Lett. 39, 78 (1984).

${ }^{7}$ G. Dresselhaus, Phys. Rev. 100, 580 (1955).

${ }^{8}$ J. Schliemann, J. C. Egues, and D. Loss, Phys. Rev. Lett. 90, 146801 (2003).

${ }^{9}$ A. A. Kiselev and K. W. Kim, Phys. Rev. B 61, 13115 (2000).

${ }^{10}$ E. Y. Sherman, Appl. Phys. Lett. 82, 209 (2003).

${ }^{11}$ M. Q. Weng, M. W. Wu, and Q. W. Shi, Phys. Rev. B 69, 125310 (2004).

${ }^{12}$ Y. V. Pershin and V. Privman, Phys. Rev. B 69, 073310 (2004).

${ }^{13}$ L. Jiang, M. Weng, M. Wu, and J. Cheng, J. Appl. Phys. 98, 113702 (2005)
${ }^{14}$ P. Schwab, M. Dzierzawa, C. Gorini, and R. Raimondi, Phys. Rev. B 74, 155316 (2006).

${ }^{15}$ M. Q. Weng, M. W. Wu, and H. L. Cui, J. Appl. Phys. 103, 063714 (2008).

${ }^{16}$ P. Kleinert and V. V. Bryksin, Phys. Rev. B 79, 045317 (2009).

${ }^{17}$ M. Duckheim, D. L. Maslov, and D. Loss, Phys. Rev. B 80, 235327 (2009).

${ }^{18}$ I. V. Tokatly and E. Y. Sherman, Ann. Phys. (NY) 325, 1104 (2010).

${ }^{19}$ M. I. Dyakonov and V. I. Perel', Sov. Phys. Solid State 13, 3023 (1972).

${ }^{20}$ A. R. Cameron, P. Riblet, and A. Miller, Phys. Rev. Lett. 76, 4793 (1996).

${ }^{21}$ E. G. Mishchenko, A. V. Shytov, and B. I. Halperin, Phys. Rev. Lett. 93, 226602 (2004).

${ }^{22}$ A. A. Burkov, A. S. Núñez, and A. H. MacDonald, Phys. Rev. B 70, 155308 (2004).

${ }^{23}$ V. M. Galitski, A. A. Burkov, and S. Das Sarma, Phys. Rev. B 74, 115331 (2006).

${ }^{24}$ S. Saikin, Y. Pershin, and V. Privman, IEE-Proc. Circ. Dev. Syst. 152, 366 (2005). 